DOI: http://dx.doi.org/10.12957/demetra.2014.9660

\title{
Ingestão alimentar de pacientes com doença celíaca no ambulatório de gastroenterologia pediátrica da Universidade Federal de Minas Gerais
}

\section{Food intake profile in pacients with celiac disease in the gastropediatric clinic of Minas Gerais Federal University, Brazil}

\author{
Marina Magalhães de Sousa-e-Silva' \\ Magda Bahia' \\ Romero Tavares Alves' \\ Francisco José Penna' \\ 1 Faculdade de Medicina, Centro de Pós \\ Graduação. Universidade Federal de Minas \\ Gerais. Belo Horizonte-MG, Brasil. \\ Correspondência /Correspondence \\ Marina Magalhães de Sousa-e-Silva \\ E-mail:marinamsousa@yahoo.com.br
}

\section{Resumo}

Introdução: A doença celíaca é uma doença autoimune, desencadeada pela presença da gliadina do trigo e de proteínas da cevada e do centeio na dieta de pessoas susceptíveis a esta disfunção, sendo o trigo uma das principais fontes de vitaminas e minerais presentes na alimentação da população. O tratamento da doença celíaca é completamente dietético, razão pela qual o acompanhamento nutricional destes indivíduos é de grande importância, pois a não adesão à dieta pelo paciente pode levar a uma deficiência nutricional. São poucos os trabalhos no Brasil que abordam os aspectos nutricionais dos pacientes com doença celíaca. Objetivo: Avaliar o perfil da ingestão alimentar de pacientes com doença celíaca. Métodos: Foram avaliados 31 pacientes com doença celíaca e 31 indivíduos saudáveis na faixa etária de três a 23 anos. A amostra foi de conveniência. Para a análise de ingestão alimentar, foi realizado um questionário de frequência alimentar, registro alimentar e recordatório durante 24 horas. Resultados: De acordo com a ingestão alimentar, o consumo de calorias e lipídeos era maior no grupo estudo. A ingestão de carboidratos era maior no grupo controle. Apesar de haver diferença na quantidade ingerida, a proporção de indivíduos que atingiam a necessidade diária para vitaminas e minerais de acordo com as IDRs selecionadas dos dois grupos não apresentou diferença estatística. Conclusão: A dieta isenta de glúten não se mostrou prejudicial ao hábito alimentar dos participantes deste estudo. A composição da dieta dos pacientes com doença celíaca se mostrou semelhante à dieta de indivíduos do grupo controle. Não foram encontradas diferenças estatísticas entre a proporção de indivíduos com ingestão ideal, abaixo ou acima de vitaminas e minerais em ambos os grupos.

Palavras-chaves: Doença Celíaca. Consumo de Alimentos. Avaliação Nutricional. 


\section{Abstract}

Introduction: Celiac disease is an autoimmune disease triggered by the presence of wheat gliadin and similar proteins in barley and rye in the diet of susceptible individuals. Since the treatment of this disease is following a gluten-free diet it is very important to keep nutritional monitoring of these individuals. Nonadherence to the diet can lead to nutritional deficiency. There are few studies in Brazil addressing the nutritional aspects of celiac disease patients. Objective: To assess the dietary intake of patients with celiac disease. Methods: We evaluated 31 patients with celiac disease and 31 healthy individuals from three to 23 years; a convenience sample was used. For analysis of food intake we carried food frequency questionnaire, dietary records and 24-hour dietary recall. Results: According to food intake, calorie and lipids consumption was higher in the study group. Carbohydrate intake was higher in the control group. Despite the difference in the amount ingested, the proportion of individuals, in both groups, who achieved the daily requirement for vitamins and minerals in accordance with the selected IDRs showed no statistical difference. Conclusion: The gluten-free diet was not harmful to the eating habits of the study participants. Diet composition of patients with celiac disease was similar to the diet of the control group. No statistical differences were found between the proportion of subjects with ideal, below or above intake for vitamins and minerals in both groups.

Key words: Celiac Disease. Nutrition Assessment. Food Consumption.

\section{Introdução}

A doença celíaca (DC) é uma doença autoimune, desencadeada pela presença da gliadina do trigo e de proteínas semelhantes da cevada e do centeio, na dieta de pessoas susceptíveis a esta enfermidade. Ela é caracterizada, principalmente, pela má absorção intestinal, devido à atrofia de vilosidades intestinais. Seus sintomas mais comuns surgem, usualmente, nos primeiros anos de vida, após a introdução desses cereais na alimentação do indivíduo. ${ }^{1}$ 
O grão do trigo é fonte de vitaminas como tiamina, niacina, riboflavina e folato, e de minerais como magnésio, potássio, ferro e selênio. A restrição deste alimento em uma dieta pode limitar a variedade de alimentos disponíveis para o consumo, sobretudo por sua importância na dieta ocidental, aliada à reduzida linha de produtos sem glúten no mercado. ${ }^{2-4}$

O tratamento multiprofissional da DC envolve uma abordagem dietética; sendo assim, é de grande importância o acompanhamento nutricional destes indivíduos, para que sua alimentação seja completa, nutritiva e isenta de glúten. A não adesão à dieta pelo paciente pode levar a deficiência nutricional, obesidade e maior incidência de neoplasias. ${ }^{5-7}$

No Ambulatório de Gastroenterologia Pediátrica do Hospital das Clínicas, da Universidade Federal de Minas Gerais (UFMG), o acompanhamento clínico é realizado com todos os pacientes diagnosticados com DC. Os casos com suspeita de distúrbios nutricionais são encaminhados para orientação através de associações de celíacos, onde também são cadastrados para controle da organização.

As crianças e adolescentes com DC podem apresentar distúrbios de composição corporal devido a hábitos alimentares inadequados e falta de orientação. Esses hábitos, especialmente em adolescentes, consistem em maior consumo de lipídeos e proteínas, já que os alimentos que são fonte de carboidrato contêm, em sua maioria, glúten. ${ }^{8}$ São poucos os trabalhos no Brasil que abordam os aspectos nutricionais dos pacientes com DC. ${ }^{7,9} \mathrm{~A}$ necessidade de monitorar o estado nutricional dos pacientes com DC e de contribuir com informações sobre este grupo populacional motivou o desenvolvimento deste projeto.

O objetivo deste estudo foi avaliar o perfil da ingestão alimentar dos pacientes com DC em tratamento no Ambulatório de Gastroenterologia Pediátrica do Hospital das Clínicas da Universidade Federal de Minas Gerais (HC-UFMG) e comparar com indivíduos da mesma faixa etária, sem a doença.

\section{Método}

Este é um estudo transversal observacional descritivo. Foi realizado no Ambulatório de Gastroenterologia Pediátrica do HC-UFMG, em datas combinadas com os indivíduos participantes da pesquisa. O grupo populacional foi constituído por pacientes com DC confirmada por biópsia intestinal, sem outras patologias associadas, atendidos no Ambulatório de Gastroenterologia Pediátrica do HC-UFMG, e voluntários associados 
da Associação dos Celíacos do Brasil de Minas Gerais (ACELBRA-MG), com faixa etária entre três e 23 anos de idade. Foram selecionados pacientes de ambos os gêneros, independentemente de raça e condição social. Participaram também indivíduos saudáveis, dentro da mesma faixa etária dos indivíduos estudados, que se voluntariaram após divulgação do projeto na UFMG e em escolas de Belo Horizonte.

O grupo estudo foi formado por pacientes com DC diagnosticada após biópsia intestinal, com faixa etária entre três e 23 anos. O grupo controle foi composto por voluntários saudáveis, com faixa etária entre três e 23 anos.

Os critérios de seleção foram: diagnóstico de DC confirmada por biópsia intestinal; e faixa etária entre três e 23 anos. Foram excluídos do estudo: pacientes com outras doenças de manifestações intestinais que não a DC; pacientes com síndrome de Down, síndrome de Turner, deficiência de IgA e deficiência de hormônio do crescimento, Diabetes Mellitus, dermatite herpetiforme; e pacientes em uso de diuréticos, devido ao protocolo para uso da bioimpedância. Os indivíduos que participaram do grupo controle atendiam aos mesmos critérios de exclusão estabelecidos para o grupo estudo.

\section{Cálculo da amostra}

A amostra foi formada por conveniência, de acordo com o fluxo de pacientes que se voluntariaram a participar da pesquisa durante o atendimento no Ambulatório de Gastroenterologia Pediátrica do HC, ou por contato através da ACELBRA-MG. A amostra final de voluntários portadores da doença foi formada por 31 crianças e adolescentes. Em um segundo momento, foram selecionados 31 voluntários saudáveis, sem a doença, com faixa etária semelhante, para compor o grupo controle.

\section{Aspectos éticos}

O projeto foi aprovado pelo Comitê de Ética e Pesquisa da UFMG no ano de 2010, parecer no ETIC 0112.0.203.000-10. Todos os participantes da pesquisa assinaram o Termo de Consentimento Livre e Esclarecido (TCLE).

\section{Coleta de dados}

A coleta de dados foi realizada no período de setembro de 2011 a junho de 2012. Os participantes foram recrutados através de ligações telefônicas realizadas pelos 
pesquisadores ou após consulta no Ambulatório de Gastroenterologia Pediátrica da Faculdade de Medicina da UFMG. A participação constava na presença em local combinado para entrevista, preenchimento de questionários e realização da antropometria.

\section{Dados coletados}

- Cálculo da Taxa Metabólica Basal (TMB), através das fórmulas propostas pela OMS. ${ }^{10}$ Cálculo do Gasto Energético Total (GET), através das referências propostas pela Sociedade Brasileira de Nutrição Parenteral e Enteral, Associação Brasileira de Cirurgia Pediatria, Sociedade Brasileira de Clínica Médica e Associação Brasileira de Nutrologia, elaborado por Coppini, Sampaio \& Marco. ${ }^{11}$ Calculados após a coleta de peso e altura do participante.

- Recordatório 24h (R24) realizado pelo pesquisador, entrevistando os indivíduos, com o auxílio de tabelas e álbum fotográfico para padronização de medidas caseiras. Esta entrevista também funcionou como uma maneira de capacitar o paciente ou seu responsável para efetuar o registro alimentar em sua própria casa. Realizado no dia da entrevista.

- Dois registros alimentares realizados em casa pelo paciente, um em dia de semana e outro em final de semana. Os registros eram entregues no dia da entrevista para serem preenchidos por um participante ou seu responsável. Após preenchimento, os registros foram enviados para o pesquisador responsável, via correio ou e-mail.

- Questionário de frequência alimentar (QFA), realizado após o R24, no dia da entrevista. Tal análise teve caráter tanto quantitativo quanto qualitativo, e o mencionado questionário foi preenchido somente uma vez.

\section{Análise de dados}

Os dados do R24 e do registro alimentar foram computados e analisados no software dietWin. Foram fornecidas informações sobre a ingestão diária média de calorias, proteínas, carboidratos e lipídeos. Os dados do QFA foram avaliados em planilha elaborada com informações de alimentos, fornecidas por tabelas de composição alimentar, como a Tabela Brasileira de Composição de Alimentos (TACO), e uma Tabela de Equivalentes, Medidas Caseiras e Composição Química dos Alimentos. ${ }^{12}$ Esta análise proveu informações sobre o consumo de colesterol, fibras totais, gordura trans, gordura saturada, vitaminas e minerais dos indivíduos. Os valores encontrados foram comparados 
com as tabelas de ingestão diária de referência (IDR) de 1998 a 2005 elaboradas pela Food and Nutrition Board ${ }^{13-17}$ da National Academies Press e por Ross et al. ${ }^{18}$

Foram utilizadas as seguintes IDRs nos nutrientes analisados: vitamina A, folato, B1, B2, B6, zinco, fósforo, magnésio, ferro, iodo (3 a 23 anos): ingestão diária recomendada (RDA); e vitamina C, D, cálcio, potássio, sódio (3 a 23 anos): ingestão adequada (AI).

O QFA foi transferido para análise em planilha do programa Excel 2010. Para que a população estudada fosse organizada por classes sociais, foi utilizado o Critério de Classificação Econômica Brasil (CCEB), elaborado pela Associação Brasileira de Empresas de Pesquisa (ABEP), em 2011. ${ }^{19}$

\section{Tratamento estatístico}

Análises descritivas foram realizadas para todas as variáveis. Foi estabelecido o grau de confiança de $95 \%$ para os testes estatísticos realizados $(\alpha=0,05)$. Para avaliar a diferença da média entre os dois grupos, foi realizado teste t para todas as variáveis normais com variância semelhante. Para as variáveis contínuas não normais, foi realizado teste de Wilcoxon. Como os dois grupos apresentam faixas etárias variadas, o que implica diferentes valores de referência para ingestão de vitaminas e minerais, as variáveis de ingestão alimentar foram categorizadas de acordo com a classificação das IDRs americanas para ingestão satisfatória, alta ou deficiente. Para estas variáveis, foi realizado o teste de Fisher, a fim de avaliar a diferença de proporções entre os dois grupos. Esse teste também foi usado para as proporções reveladas pelo questionário

socioeconômico. O teste de Pearson foi aplicado nas variáveis contínuas de interesse, a fim de avaliar a correlação entre as amostras. Os testes foram executados por meio dos programas Microsoft Excel, R e IBM SPSS Statistics.

\section{Resultados}

\section{Avaliação socioeconômica}

Sessenta e dois participantes foram avaliados no período de estudo, divididos em dois grupos de 31 indivíduos cada. É possível observar, na tabela 1, as informações sobre a distribuição dos participantes dos dois grupos em relação a gênero, cor da pele, classe social, acompanhamento nutricional e prática de atividades físicas. Não foi encontrada diferença estatística nestes parâmetros, somente na classificação social $(p=0,00)$, podendo indicar diferença de poder aquisitivo entre os dois grupos. Nas figuras 1 e 2 , observa-se a 
distribuição dos grupos de acordo com a faixa etária. As médias encontradas para o grupo estudo (13 anos $\pm 5,79)$ e para o grupo controle $(13,42$ anos $\pm 5,70)$ são semelhantes, de acordo com o teste $\mathrm{t}(\mathrm{p}=0,39)$.

Tabela 1. Distribuição dos grupos estudo e controle de acordo com informações do questionário socioeconômico - Ambulatório de Gastroenterologia Pediátrica da UFMG. Belo Horizonte-MG, junho de 2012.

\begin{tabular}{|c|c|c|c|c|c|}
\hline \multirow[b]{2}{*}{ Gênero } & \multicolumn{2}{|c|}{ Grupo Estudo } & \multicolumn{2}{|c|}{ Grupo Controle } & \multirow[t]{2}{*}{$\mathrm{P}$} \\
\hline & $\mathrm{n}$ & $\%$ & $\mathrm{n}$ & $\%$ & \\
\hline Masculino & 23 & 74,19 & 21 & 67,74 & \multirow[t]{2}{*}{0,78} \\
\hline Feminino & 8 & 25,81 & 10 & 32,26 & \\
\hline Cor da pele & $\mathrm{n}$ & $\%$ & $\mathrm{n}$ & $\%$ & \\
\hline Branca & 21 & 67,74 & 18 & 58,06 & \multirow{4}{*}{0,19} \\
\hline Parda & 8 & 25,81 & 13 & 41,94 & \\
\hline Negra & 2 & 6,45 & 0,00 & 0,00 & \\
\hline Outras & 0 & 0,00 & 0,00 & 0,00 & \\
\hline Classe Social & $\mathrm{n}$ & $\%$ & $\mathrm{n}$ & $\%$ & \\
\hline $\mathrm{A} 1(\mathrm{R} \$ 11.480,00)$ & 1 & 3,23 & 5 & 16,13 & \multirow{6}{*}{0,00} \\
\hline $\mathrm{A} 2(\mathrm{R} \$ 8.295,00)$ & 3 & 9,68 & 16 & 51,61 & \\
\hline $\mathrm{B} 1(\mathrm{R} \$ 4.754,00)$ & 7 & 22,58 & 5 & 16,13 & \\
\hline $\mathrm{B} 2(\mathrm{R} \$ 2.656,00)$ & 13 & 41,94 & 1 & 3,23 & \\
\hline $\mathrm{C} 1(\mathrm{R} \$ 1.459,00)$ & 4 & 12,90 & 4 & 12,90 & \\
\hline $\mathrm{C} 2(\mathrm{R} \$ 962,00)$ & 3 & 9,68 & 0 & 0,00 & \\
\hline Acompanhamento nutricional & $\mathrm{n}$ & $\%$ & $\mathrm{n}$ & $\%$ & \\
\hline Sim & 12 & 38,71 & 5 & 16,13 & \multirow[t]{2}{*}{0,08} \\
\hline Não & 19 & 61,29 & 26 & 83,87 & \\
\hline Prática de Atividade física & $\mathrm{n}$ & $\%$ & $\mathrm{n}$ & $\%$ & \multirow{3}{*}{0,19} \\
\hline Sim & 15 & 48,39 & 21 & 67,74 & \\
\hline Não & 16 & 51,61 & 10 & 32,26 & \\
\hline
\end{tabular}




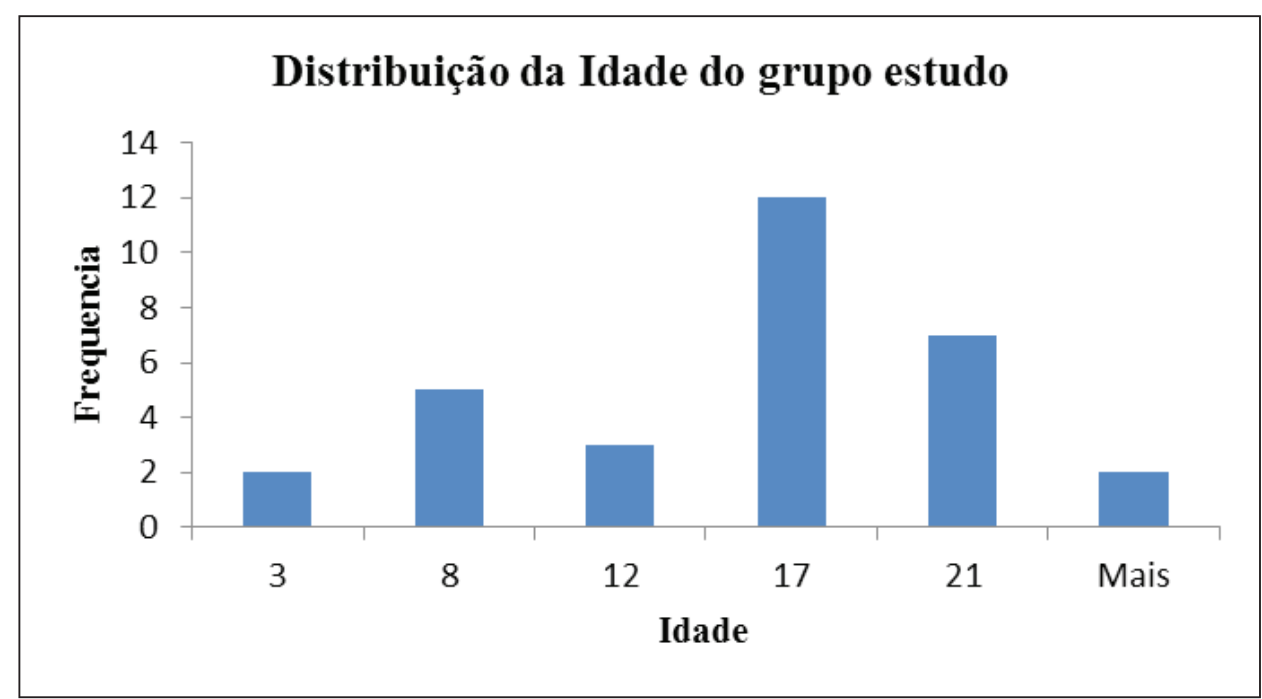

Figura 1. Distribuição dos participantes do grupo estudo de acordo com a idade - Ambulatório de Gastroenterologia Pediátrica da UFMG. Belo Horizonte-MG, junho de 2012.

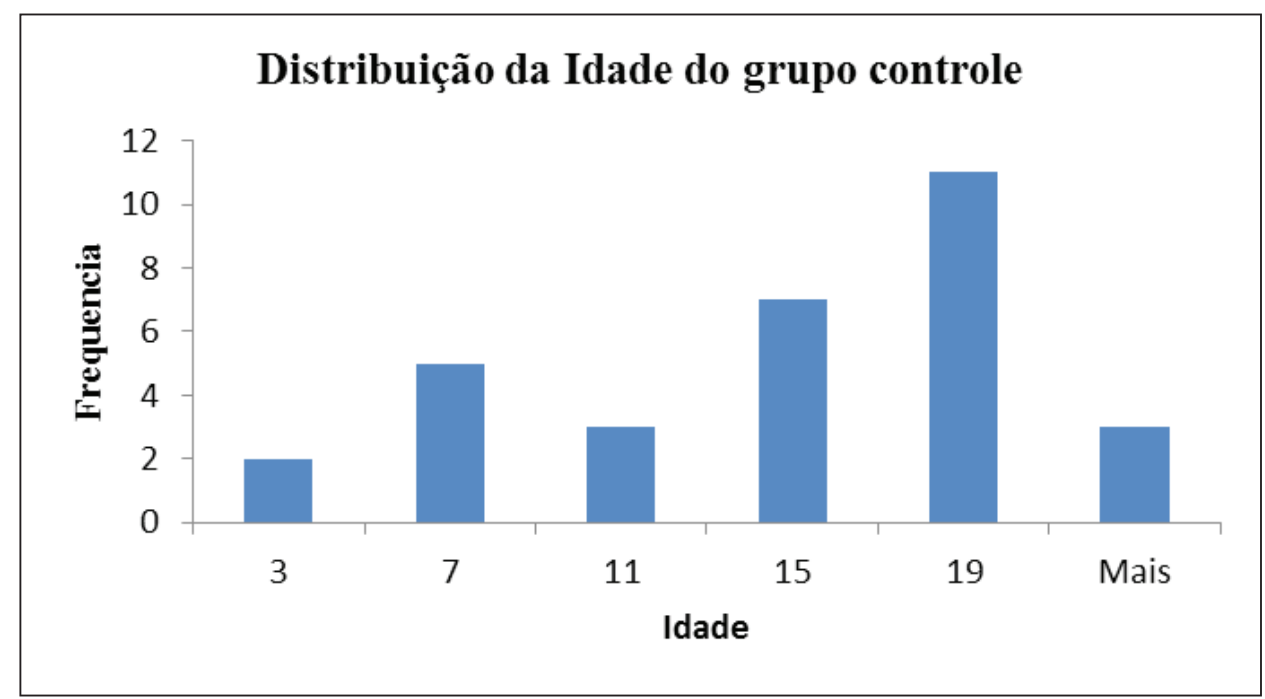

Figura 2. Distribuição dos participantes do grupo controle de acordo com a idade. Ambulatório de Gastroenterologia Pediátrica da UFMG. Belo Horizonte-MG, junho de 2012. 
O grupo estudo respondeu a uma parte do questionário relacionada somente a DC e seus sintomas. Cem por cento dos participantes apresentavam biópsia intestinal como diagnóstico principal para a DC e realizaram ao menos um teste sorológico para DC. No mesmo grupo, 77,42\% dos pacientes admitiam seguir dieta completamente isenta de glúten.

Em relação aos sintomas pré-diagnóstico da doença, 74,19\% dos participantes relataram apresentar algum tipo de manifestação gastrointestinal, em sua maioria diarreia $(51,61 \%)$, seguida de vômitos $(19,35 \%)$, perda de peso $(16,13 \%)$ e desconforto abdominal $(16,13 \%)$.

\section{Ingestão de macronutrientes}

De acordo com as informações apresentadas no figura 1, o consumo diário de arroz e feijão, que são alimentos frequentes na refeição do brasileiro, foi maior entre os participantes do grupo estudo (30 e 29 indivíduos contra 28 e 22, respectivamente do grupo controle). O grupo controle apresentou menor consumo diário de carnes (18 indivíduos contra 24), alimentos industrializados (23 contra 16) e embutidos (7 contra 2). O grupo denominado "Pães de queijo e biscoito de polvilho" foi escolhido devido ao grande consumo destes alimentos pelos indivíduos portadores de DC, justamente por serem isentos de glúten. Esses dados foram revelados com fins meramente ilustrativos, sem realização de testes estatísticos, por não serem foco de análise do trabalho. 


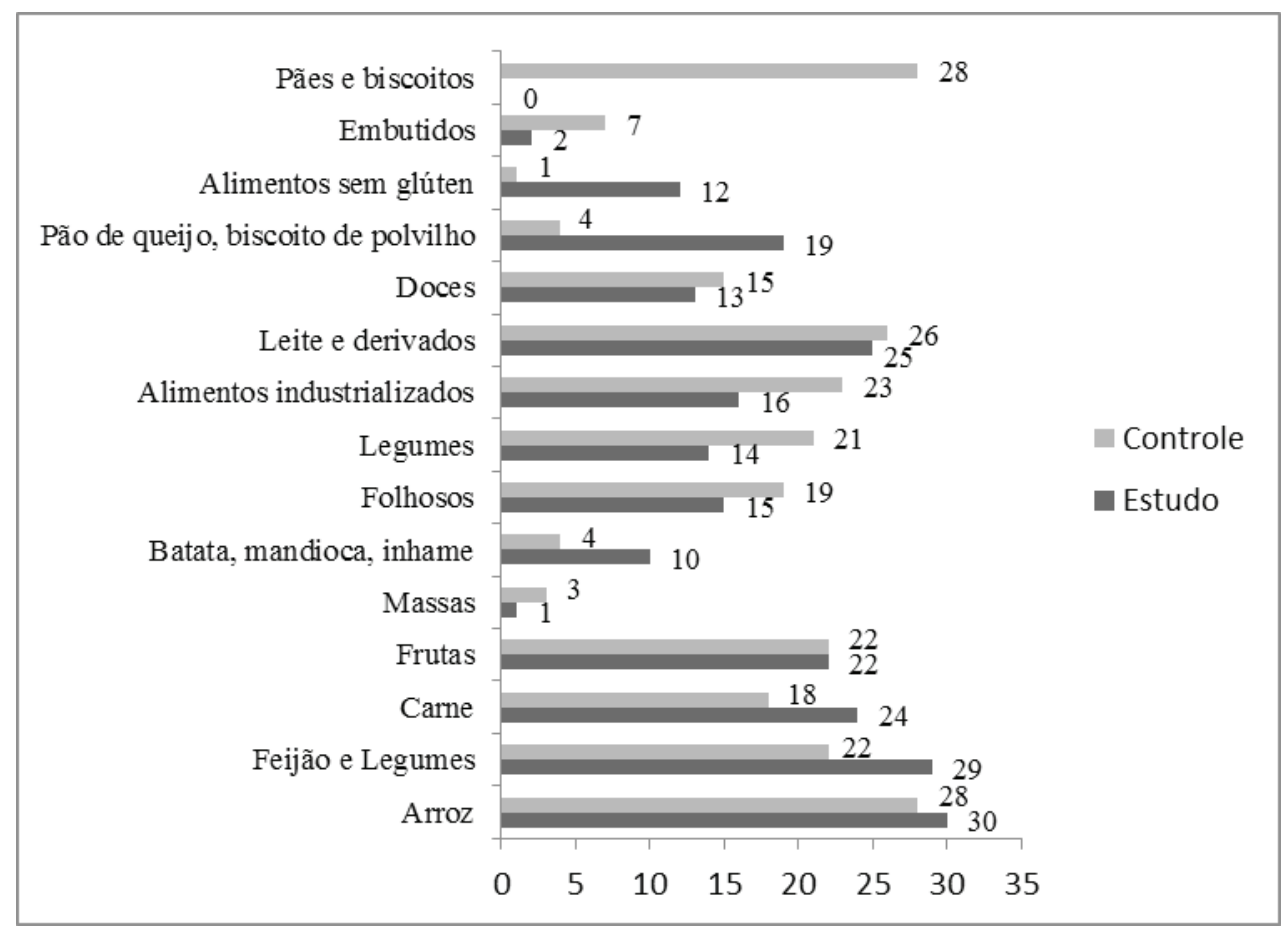

Figura 3. Consumo alimentar diário dos participantes do estudo de acordo com o questionário de frequência alimentar. Ambulatório de Gastroenterologia Pediátrica da UFMG. Belo Horizonte-MG, junho de 2012.

Efetuada a separação dos alimentos em grupos alimentares de acordo com a pirâmide alimentar proposta por Welsh, Davis \& Shaw, ${ }^{20} \mathrm{em} 1992$, nos Estados Unidos, observouse que o consumo diário de cereais, tubérculos e raízes, que são a base da pirâmide, é semelhante entre o grupo estudo e o grupo controle. Há diferença no consumo de carnes e ovos, que é menor no grupo estudo, e leguminosas, que é menor no grupo controle. $\mathrm{O}$ teste de Fisher não revelou diferença estatística entre os dois grupos com relação à ingestão de grupos alimentares, de acordo com a pirâmide alimentar.

Segundo a tabela 2, a média de ingestão calórica diária dos indivíduos do grupo estudo é estatisticamente maior que a do grupo controle. Observou-se, ainda, que o gasto energético total médio dos dois grupos é semelhante $(\mathrm{p}>0,05)$. Pode-se verificar ainda que é maior, no grupo estudo, o número de pacientes com consumo calórico acima do GET diário calculado para cada indivíduo (29 indivíduos do grupo estudo contra 22 do grupo controle). Essas informações, por serem consideradas complementares, não estão apresentadas em tabelas, e não foram realizados testes estatísticos. 
Tabela 2. Análises descritivas dos valores diários encontrados para o valor calórico total (VCT) das dietas, de acordo com o recordatório 24h, e do gasto energético total (GET) dos grupos estudo e controle. Ambulatório de Gastroenterologia Pediátrica da UFMG. Belo Horizonte-MG, junho de 2012.

\begin{tabular}{c|c|c|c|c|c|c}
\hline \multirow{2}{*}{ Variável } & \multicolumn{2}{|c|}{ VCT R24 } & P & \multicolumn{2}{|c|}{ GET (Kcal) } & P \\
\cline { 2 - 6 } & Estudo & Controle & & Estudo & Controle & \\
Mediana & $2.619,77$ & $2.017,10$ & & $1.872,83$ & $1.832,70$ & \\
Média & $2.602,05$ & $2.001,40$ & & $1.831,09$ & $1.949,37$ & \\
DP* & 703,89 & 619,17 & 0,00 & 598,01 & 549,42 & 0,25 \\
CV $\dagger$ & 0,27 & 0,31 & & 0,33 & 0,28 & \\
\hline
\end{tabular}

†Legenda: (*) Desvio-padrão (†) Coeficiente de Variância

De acordo com a tabela 3, o consumo médio, em gramas, de carboidratos e lipídeos, é significativamente maior no grupo estudo. O consumo médio proteico é maior no grupo controle, porém sem significância estatística.

Tabela 3. Análises descritivas da ingestão, em gramas, de CHO, LIP e PTN dos grupos estudo e controle de acordo com o recordatório 24h. Belo Horizonte-MG, 2012.

\begin{tabular}{|c|c|c|c|c|c|c|c|c|c|}
\hline \multirow{2}{*}{ Estatísticas } & \multicolumn{2}{|c|}{$\mathrm{CHO}(\mathrm{g})$} & $\mathrm{p}$ & \multicolumn{2}{|c|}{ PTN (g) } & $\mathrm{p}$ & \multicolumn{2}{|c|}{ LIP (g) } & $\mathrm{P}$ \\
\hline & Estudo & Controle & & Estudo & Controle & & Estudo & Controle & \\
\hline Mediana & 369,82 & 248,75 & & 98,99 & 78,98 & & 76,01 & 61,21 & \\
\hline Média & 371,02 & 266,07 & & 93,45 & 94,20 & & 78,73 & 61,02 & \\
\hline $\mathrm{DP}^{*}$ & 127,09 & 97,96 & 0,00 & 33,62 & 57,14 & 0,41 & 24,59 & 21,60 & 0,00 \\
\hline $\mathrm{CV} \dagger$ & 0,34 & 0,37 & & 0,36 & 0,61 & & 0,31 & 0,35 & \\
\hline
\end{tabular}

†Legenda: (*) Desvio-padrão (†) Coeficiente de Variância 


\section{Ingestão de vitaminas e minerais}

Aplicado o teste de Fischer para as variáveis de vitaminas categorizadas, conforme exposto na tabela 4, observou-se que não houve diferença de proporção de participantes com ingestão ideal de vitaminas, abaixo ou acima do recomendado, entre os dois grupos. Da mesma forma, conforme a aplicação do teste de Fischer para a ingestão de minerais e fibras totais categorizadas de acordo com as IDRs, observou-se que não houve diferença estatisticamente significativa para as proporções de ingestão ideal, abaixo ou acima do recomendado para os dois grupos. Estas semelhanças, entretanto, não afirmam que $o$ perfil de ingestão alimentar encontrado em ambos os grupos é o ideal, de acordo com as referências internacionais.

Tabela 4. Percentual de indivíduos dos grupos estudo e controle que atingiram, ou não, as IDRs para vitaminas, minerais e fibras totais de acordo com o QFA. Ambulatório de Gastroenterologia Pediátrica da UFMG. Belo Horizonte-MG, junho de 2012.

\begin{tabular}{|c|c|c|c|c|c|}
\hline \multirow{3}{*}{ Nutriente } & \multicolumn{5}{|c|}{ Grupos } \\
\hline & \multicolumn{2}{|c|}{ Estudo } & \multicolumn{2}{|c|}{ Controle } & \multirow[b]{2}{*}{$\mathrm{p}$} \\
\hline & $\mathrm{n}$ & $\%$ & $\mathrm{n}$ & $\%$ & \\
\hline \multicolumn{6}{|l|}{ Vitamina A } \\
\hline Abaixo & 10 & 32,26 & 10 & 32,26 & \\
\hline Adequado & 21 & 67,74 & 21 & 67,74 & 1 \\
\hline Acima & 0 & 0 & 0 & 0 & \\
\hline \multicolumn{6}{|l|}{ Vitamina D } \\
\hline Abaixo & 21 & 67,74 & 17 & 54,84 & \\
\hline Adequado & 10 & 32,26 & 14 & 45,16 & 0,43 \\
\hline Acima & 0 & 0 & 0 & 0 & \\
\hline \multicolumn{6}{|l|}{ Vitamina E } \\
\hline Abaixo & 3 & 9,68 & 2 & 6,45 & \\
\hline Adequado & 28 & 90,32 & 29 & 93,55 & 1 \\
\hline Acima & 0 & 0 & 0 & 0 & \\
\hline
\end{tabular}




\begin{tabular}{|c|c|c|c|c|c|}
\hline \multicolumn{6}{|l|}{ Vitamina B1 } \\
\hline Abaixo & 0 & 0 & 1 & 3,23 & \multirow{3}{*}{1} \\
\hline Adequado & 31 & 100 & 30 & 96,77 & \\
\hline Acima & 0 & 0 & 0 & 0 & \\
\hline \multicolumn{6}{|l|}{ Vitamina B2 } \\
\hline Abaixo & 9 & 29,03 & 4 & 12,9 & \multirow{3}{*}{1} \\
\hline Adequado & 22 & 70,97 & 27 & 87,1 & \\
\hline Acima & 0 & 0 & 0 & 0 & \\
\hline \multicolumn{6}{|l|}{ Vitamina B6 } \\
\hline Abaixo & 2 & 6,45 & 2 & 6,45 & \multirow{3}{*}{1} \\
\hline Adequado & 29 & 93,55 & 29 & 93,55 & \\
\hline Acima & 0 & 0 & 0 & 0 & \\
\hline \multicolumn{6}{|l|}{ Folato } \\
\hline Abaixo & 26 & 83,87 & 16 & 51,61 & \multirow{3}{*}{0,45} \\
\hline Adequado & 5 & 16,13 & 15 & 48,39 & \\
\hline Acima & 0 & 0 & 0 & 0 & \\
\hline \multicolumn{6}{|l|}{ Vitamina B12 } \\
\hline Abaixo & 5 & 16,13 & 6 & 19,35 & \multirow{3}{*}{1} \\
\hline Adequado & 26 & 83,87 & 25 & 80,65 & \\
\hline Acima & 0 & 0 & 0 & 0 & \\
\hline \multicolumn{6}{|l|}{ Vitamina $\mathrm{C}$} \\
\hline Abaixo & 5 & 16,13 & 2 & 6,45 & \multirow{3}{*}{1} \\
\hline Adequado & 26 & 83,87 & 29 & 93,55 & \\
\hline Acima & 0 & 0 & 0 & 0 & \\
\hline \multicolumn{6}{|l|}{ Cálcio } \\
\hline Abaixo & 13 & 41,94 & 20 & 64,52 & \multirow{3}{*}{0,12} \\
\hline Adequado & 18 & 58,06 & 11 & 35,48 & \\
\hline Acima & 0 & 0 & 0 & 0 & \\
\hline
\end{tabular}




\begin{tabular}{|c|c|c|c|c|c|}
\hline Ferro & & & & & \\
\hline Abaixo & 6 & 19,35 & 12 & 38,71 & \multirow{3}{*}{0,16} \\
\hline Adequado & 25 & 80,65 & 19 & 61,29 & \\
\hline Acima & 0 & 0 & 0 & 0 & \\
\hline \multicolumn{6}{|l|}{ Sódio } \\
\hline Abaixo & 0 & 0 & 0 & 0 & \multirow{3}{*}{0,17} \\
\hline Adequado & 7 & 22,58 & 13 & 41,94 & \\
\hline Acima & 24 & 77,42 & 18 & 58,06 & \\
\hline \multicolumn{6}{|l|}{ Potássio } \\
\hline Abaixo & 24 & 77,42 & 26 & 83,87 & \multirow{3}{*}{0,74} \\
\hline Adequado & 7 & 22,58 & 5 & 16,13 & \\
\hline Acima & 0 & 0 & 0 & 0 & \\
\hline \multicolumn{6}{|l|}{ Zinco } \\
\hline Abaixo & 8 & 25,81 & 13 & 41,94 & \multirow{3}{*}{0,28} \\
\hline Adequado & 23 & 74,19 & 18 & 58,06 & \\
\hline Acima & 0 & 0 & 0 & 0 & \\
\hline \multicolumn{6}{|l|}{ Fósforo } \\
\hline Abaixo & 7 & 22,58 & 13 & 41,94 & \multirow{3}{*}{0,17} \\
\hline Adequado & 24 & 77,42 & 18 & 58,06 & \\
\hline Acima & 0 & 0 & 0 & 0 & \\
\hline \multicolumn{6}{|l|}{ Magnésio } \\
\hline Abaixo & 22 & 70,97 & 24 & 77,42 & \multirow{3}{*}{0,77} \\
\hline Adequado & 9 & 29,03 & 7 & 22,58 & \\
\hline Acima & 0 & 0 & 0 & 0 & \\
\hline \multicolumn{6}{|l|}{ Iodo } \\
\hline Abaixo & 23 & 74,19 & 20 & 64,52 & \multirow{3}{*}{0,58} \\
\hline Adequado & 8 & 25,81 & 11 & 35,48 & \\
\hline Acima & 0 & 0 & 0 & 0 & \\
\hline
\end{tabular}




\begin{tabular}{c|c|c|c|c|c}
\hline \multicolumn{2}{l|}{ Fibras Totais } \\
\cline { 1 - 4 } Abaixo & 21 & 67,74 & 24 & 77,42 & \multirow{2}{*}{0,57} \\
Adequado & 10 & 32,26 & 7 & 22,58 & \\
\hline Acima & 0 & 0 & 0 & 0 & \\
\hline
\end{tabular}

\section{Discussão}

De acordo com os resultados encontrados neste estudo, os participantes do grupo estudo e grupo controle, quando investigado o perfil de ingestão de macro e micronutrientes, podem ser considerados semelhantes. Algumas diferenças nos hábitos alimentares foram encontradas, mas não refletiram em fornecimento difereciado de nutrientes no saldo final. A ingestão alimentar dessas crianças e adolescentes é deficitária em muitos pontos, mas neste estudo, tal fato não é relacionado com a presença da doença celíaca.

Alguns autores, como Mariani et al., ${ }^{8}$ relatam que a criança e, com mais frequência, o adolescente, têm habitualmente maior dificuldade em seguir padrões saudáveis de alimentação, independentemente da presença de alguma doença. Fonseca, Sichieri \& Veiga $^{21}$ reforçam que o hábito alimentar do adolescente consiste, principalmente, no consumo de preparações calóricas, omissão de refeições como o desjejum e a prática de refeições rápidas. Estes hábitos, associados à baixa prática de atividades físicas, aumentam a prevalência de sobrepeso e obesidade para este grupo etário. ${ }^{21}$ Carvalho, Oliveira \& Santos $^{22}$ encontraram baixa ingestão de frutas, legumes, leite e verduras, além de alto consumo de balas, chicletes, alimentos fritos, chips e refrigerante em crianças de uma escola municipal de Belo Horizonte. Os autores associam este perfil de ingestão alimentar à transição nutricional brasileira nas últimas três décadas. ${ }^{22}$

Essas descrições do perfil alimentar, encontradas nesta faixa etária, reforçam que as deficiências nutricionais podem não estar relacionadas com a presença da doença celíaca. No presente estudo, foi possível perceber um grande consumo de produtos açucarados, como refrigerantes, doces e biscoitos, em ambos os grupos, através da análise dos registros alimentares e QFA. No entanto, este consumo era maior entre os participantes do grupo controle. Os indivíduos não portadores de DC também consumiam maior quantidade de embutidos e alimentos industrializados. 
Dall'Asta et al. ${ }^{23}$ encontraram maior ingestão de doces e refrigerantes em pacientes celíacos, quando comparados com o grupo controle, porém os pacientes estudados eram adultos, o que poderia explicar a diferença de perfil alimentar quando comparado com o grupo avaliado neste estudo: os pacientes jovens sofrem maior influência dos pais para seguir uma dieta nutricionalmente adequada, especialmente se eles já apresentam algum tipo de deficiência nutricional. ${ }^{7,24}$

Os indivíduos do grupo estudo apresentavam menor consumo diário de proteínas, vegetais e frutas, porém maior consumo de arroz, feijão, batata e outras raízes e tubérculos. $\mathrm{O}$ fato de apresentarem menor renda familia mensal que o grupo controle, e consequente menor gasto com alimentação, poderia estar associado com a redução no consumo de carnes, vegetais, frutas e produtos industrializados, que são mais caros.

Observou-se que os indivíduos com DC ingeriam, em sua maioria, quantidade maior de calorias do que seu gasto energético total (GET). A quantidade de indivíduos com consumo calórico maior que o GET no grupo estudo (29) era maior que no grupo controle (22). No entanto, não houve diferença estatística entre as médias de gasto energético total e taxa metabólica basal dos dois grupos.

A maior ingestão calórica diária total, encontrada no grupo estudo quando comparada com seu grupo controle, pode ser uma característica da dieta destes pacientes. Em estudo realizado com pacientes já na idade adulta, Thompson et al. ${ }^{25}$ encontraram ingestão calórica média diária elevada em um com DC, concordando com o perfil encontrado neste estudo, apesar da diferença de faixa etária. Cheng et al., ${ }^{26}$ Valleta et al., ${ }^{27}$ Reilly et al. ${ }^{28}$ e Dickey e Kearney ${ }^{6}$ discutem, em seus trabalhos, a possibilidade do aumento do consumo calórico após o início de DIG já que, nos três estudos, foi encontrado aumento do peso e do IMC após retirada do glúten da alimentação. O aumento da ingestão calórica acontece independentemente do sexo ou da faixa etária estudada.

Apesar destes indícios, os participantes do grupo estudo e controle, neste trabalho, se assemelharam, em relação à composição da dieta, no que se refere ao consumo de macronutrientes. O percentual médio do consumo de carboidratos e lipídeos de ambos os grupos é semelhante, sendo que somente o percentual médio de consumo proteico é menor no grupo estudo. Alguns trabalhos, como o de Mariani et al., ${ }^{8}$ encontraram que a dieta dos pacientes celíacos era hiperlipídica e hiperproteica, associada à baixa ingestão de carboidratos. Polito et al. ${ }^{29}$ também encontraram alto consumo de proteínas e lipídeos em crianças que seguiam DIG. Uma variável que pode ser importante, nos diferentes perfis encontrados em crianças e adolescentes celíacos de nosso estudo, é a baixa renda familiar, que não permitiria o alto consumo de alimentos proteicos e gordurosos com frequência, como já foi discutido anteriormente. 
De acordo com o teste de Fisher, realizado para avaliar diferenças entre as proporções de pacientes com ingestão de vitaminas ideal ou abaixo do recomendado, não houve diferença estatística entre os dois grupos no que se refere ao consumo destes nutrientes. Malandrino et al. ${ }^{30}$ afirmam que tais deficiências são encontradas apenas quando ocorre transgressão da dieta, e que a presença do glúten lesa a mucosa intestinal, dificultando a absorção de vitaminas e mineirais. No presente estudo, a taxa de transgressão da dieta, em pacientes com DC, era baixa e ocasional. Malandrino at al. ${ }^{30}$ comentam ainda que a dieta isenta de glúten não é nutricionalmente inadequada, apesar de diminuir a ingestão de fibras alimentares por causa da restrição de produtos integrais.

A ingestão de fibras alimentares é reduzida em ambos os grupos. Apesar da baixa disponibilidade da fibra na DIG, devido também ao uso de farinhas refinadas com poucos nutrientes, além de redução de carboidrato para produção de alimentos sem glúten, o consumo de fibras é reduzido nos jovens brasileiros. De acordo com a Pequisa de Orçamentos Familiares realizada pelo IBGE ${ }^{31}$ em 2008 e 2009, 82\% dos indivíduos do sexo feminino e $78 \%$ dos indivíduos do sexo masculino, de 10 a 13 anos, não atingem a meta proposta para ingestão de fibras alimentares pelo Ministério da Saúde do Brasil. Estes valores se mantêm na faixa etária de 14 a 18 anos, na qual $77 \%$ dos indivíduos masculinos e $86 \%$ do sexo feminino estão inadequados quanto à ingestão de fibras alimentares.

\section{Conclusão}

No presente estudo, após avaliação da ingestão alimentar, não foi encontrada diferença nas proporções de indivíduos classificados com ingestão ideal, abaixo ou acima do recomendado pelas IDRs americanas.

A composição da dieta dos pacientes com DC se mostrou semelhante e, em alguns itens, mais adequada do que a de indivíduos do grupo controle. Apesar de se queixarem da dificuldade de seguir a dieta isenta de glúten, os pacientes com DC têm boa adesão a este tratamento, por estarem registrados em uma associação de doentes celíacos. As adaptações dietéticas realizadas por estes indivíduos não revelaram ser o motivo de carência nutricional quando avaliada a ingestão de alguns minerais ou vitaminas.

O perfil da ingestão alimentar do grupo estudado é associado à faixa etária dos participantes, que apresentam maior consumo calórico e baixa adesão a produtos naturais, e não ao quadro clínico dos pacientes com DC e sua observância à dieta isenta de glúten. 
A diferença de poder aquisitivo entre os dois grupos apresentados neste estudo pode ter papel importante nas diferenças de variáveis avaliadas em ambos os grupos, bem como a prática de atividade física e o consumo de proteínas. No entanto, esses fatores não resultaram em diferença no perfil nutricional dos indivíduos aqui avaliados.

\section{Agradecimentos}

À ACELBRA-MG, por todo apoio durante o período da pesquisa.

\section{Referências}

1. Kagnoff MF. Overview and pathogenesis of celiac disease. Gastroenterology. 2005; 128(4 Suppl 1):S10-8.

2. Thompson T. Thiamin, riboflavin, and niacin contents of the gluten-free diet: is there cause for concern? J. Am. Diet Assoc. 1999; 99(7):858-62.

3. Lee A, Newman JM. Celiac diet: its impact on quality of life. J. Am. Diet Assoc. 2003; 103(11):1533-5.

4. Carneiro H. Comida e sociedade. Rio de Janeiro: Campus; 2003.

5. Butterworth JR, Banfield LM, Iqbal TH, Cooper BT. Factors relating to compliance with a glutenfree diet in patients with coeliac disease: comparison of white Caucasian and South Asian patients. Clin. Nutr. 2004; 23(5):1127-34.

6. Dickey W, Kearney N. Overweight in celiac disease: prevalence, clinical characteristics, and effect of a gluten-free diet. Am. J. Gastroenterol. 2006; 101(10):2356-9.

7. Sdepanian VL, Morais MB, Fagundes-Neto U. Celiac disease: evaluation of compliance to gluten-free diet and knowledge of disease in patients registered at the Brazilian Celiac Association (ACA). Arq. Gastroenterol. 2001; 38(4):232-9.

8. Mariani P, Viti MG, Montuori M, La Vecchia A, Cipolletta E, Calvani L, et al. The gluten-free diet: a nutritional risk factor for adolescents with celiac disease? J. Pediatr. Gastroenterol. Nutr. 1998; 27(5):519-23.

9. Araújo HMC. Impacto da doença celíaca na saúde, práticas alimentares e na qualidade de vida de celíacos [Dissertação]. Brasília: Universidade de Brasília; 2008.

10. World Health Organization. Energy and protein requirements. Report of a joint FAO/WHO/UNU expert consultation. Geneva: World Health Organization; 1985. World Health Organization Technical Report Series 724.

11. Coppini LZ, Sampaio H, Marco D. Recomendações nutricionais para crianças em terapia nutricional enteral e parenteral. In: Jatene FB, Bernardo WM. Projeto diretrizes: Associação Médica Brasileira e Conselho Federal de Medicina. v. 9. Brasília: Conselho Federal de Medicina; 2011. p. 35-50. 
12. Pacheco M. Tabela de equivalentes, medidas caseiras e composição química dos alimentos. São Paulo: Rúbio; 2011.

13. Food and Nutrition Board. Dietary reference intakes for energy, carbohydrate, fiber, fat, fatty acids, cholesterol, protein and amino acids. Washington, D.C.: The National Academies Press; 2005. 1357 p.

14. Food and Nutrition Board. Dietary reference intakes for thiamin, riboflavin, niacin, vitamin b6, folate, vitamin b12, pantothenic acid, biotin and choline. Washington, D.C.: The National Academies Press; 1998. 592 p.

15. Food and Nutrition Board. Dietary reference intakes for vitamin a, vitamin k, arsenic, boron, chromium, copper, iodine, iron, manganese, molybdenium, nickel, silicon, vanadium and zinc. Washington, D.C.: The National Academies Press; 2001. 800 p.

16. Food and Nutrition Board. Dietary Reference Intakes for Vitamin C, Vitamin E, Selenium and Carotenoids. The National Academies Press; 2000. 529p.

17. Food and Nutrition Board. Dietary Reference Intakes for Water, Potassium, Sodium, Chloride and Sulfate. The National Academies Press; 2005. 640p.

18. Ross CA, Taylor CL, Yaktine AL, Valle HB. Dietary Reference Intakes for Calcium and Vitamin D. Washington: The National Academies Press; 2011.

19. Associação Brasileira de Empresas de Pesquisa. Critério de classificação econômica Brasil [Internet]. São Paulo: ABEP. [acesso em dez. 2011]. Disponível em: http://www.abep.org/new/criterioBrasil.aspx

20. Welsh S, Davis C, Shaw A. Development of the food guide pyramid. Nutr. Today 1992; 27(6):12-23.

21. Fonseca VM, Sichieri R, Veiga GV. Factors associated with obesity among adolescents. Rev. Saude Publica. 1998; 32(6):541-9.

22. Carvalho AP, Oliveira VB, Santos LC. Hábitos alimentares e práticas de educação nutricional: atenção a crianças de uma escola municipal de Belo Horizonte, Minas Gerais. Pediatria (São Paulo). 2010; 32(1):20-7.

23. Dall'Asta C, Scarlato AP, Galaverna G, Brighenti F, Pellegrini N. Dietary exposure to fumonisins and evaluation of nutrient intake in a group of adult celiac patients on a gluten-free diet. Mol. Nutr. Food Res. 2012; 56(4):632-40.

24. Greco L, Mayer M, Ciccarelli G, Troncone R, Auricchio S. Compliance to a gluten-free diet in adolescents, or "what do 300 coeliac adolescents eat every day?". Ital. J. Gastroenterol. Hepatol. 1997; 29(4):305-10.

25. Thompson T, Dennis M, Higgins LA, Lee AR, Sharrett MK. Gluten-free diet survey: are Americans with coeliac disease consuming recommended amounts of fibre, iron, calcium and grain foods? J. Hum. Nutr. Diet. 2005; 18(3):163-9.

26. Cheng J, Brar PS, Lee AR, Green PH. Body mass index in celiac disease: beneficial effect of a glutenfree diet. J. Clin. Gastroenterol. 2010; 44(4):267-71.

27. Valletta E, Fornaro M, Cipolli M, Conte S, Bissolo F, Danchielli C. Celiac disease and obesity: need for nutritional follow-up after diagnosis. Eur. J. Clin. Nutr. 2010; 64(11):1371-2. 
28. Reilly NR, Aguilar K, Hassid BG, Cheng J, Defelice AR, Kazlow P, et al. Celiac disease in normalweight and overweight children: clinical features and growth outcomes following a gluten-free diet. J. Pediatr. Gastroenterol. Nutr. 2011; 53(5):528-31.

29. Polito C, Olivieri AC, Marchese L, Desiderio G, Pullano F, Rea F. Weight overgrowth of coeliac children on gluten-free diet. Nutr. Res. 1992; 12(3):353-8.

30. Malandrino N, Capristo E, Farnetti S, Leggio L, Abenavoli L, Addolorato G, et al. Metabolic and nutritional features in adult celiac patients. Dig Dis. 2008; 26(2):128-33.

31. Instituto Brasileiro de Geografia e Estatística. Pesquisa de orçamentos familiares 2008-2009: análise do consumo alimentar pessoal no Brasil. Rio de Janeiro: IBGE; 2011. 150 p.

Recebido: 28/2/2014

Revisado: 03/7/2014

Aprovado: 18/8/2014 